\title{
MicroRNAs sequencing unveils distinct molecular subgroups of plasmablastic Iymphoma
}

\author{
Maria Raffaella Ambrosio ${ }^{1, *}$, Lucia Mundo ${ }^{1, *}$, Sara Gazaneo ${ }^{1}$, Matteo Picciolini ${ }^{2}$, \\ Prasad Satya Vara ${ }^{3}$, Shaheen Sayed ${ }^{4}$, Alessandro Ginorii,5, Giuseppe Lo Bello ${ }^{1}$, \\ Leonardo Del Porro ${ }^{1}$, Mohsen Navari $6,7,8$, Stefano Ascani ${ }^{9}$, Amhed Yonis ${ }^{10}$, Lorenzo \\ Leoncini $^{1}$, Pier Paolo Piccaluga ${ }^{7,8, *}$ and Stefano Lazzi ${ }^{1, *}$ \\ ${ }^{1}$ Department of Medical Biotechnology, Section of Pathology, University of Siena, Siena, Italy \\ 2 Diatech Pharmacogenetics, Jesi, Italy \\ ${ }^{3}$ Aga Khan Hospital, Kisumu, Kenya \\ ${ }^{4}$ Aga Khan University Hospital, Nairobi, Kenya \\ ${ }^{5}$ Pathology Unit, Ospedale Civico di Carrara, Carrara, Italy \\ ${ }^{6}$ Research Center of Advanced Technologies in Medicine, Torbat Heydariyeh University of Medical Sciences, Torbat Heydariyeh, \\ Iran \\ ${ }^{7}$ Department of Experimental, Diagnostic, and Experimental Medicine, Bologna University School of Medicine, Bologna, Italy \\ ${ }^{8}$ Euro-Mediterranean Institute of Science and Technology (IEMEST), Palermo, Italy \\ ${ }^{9}$ Section of Pathology, Azienda Ospedaliera S. Maria di Terni, University of Perugia, Perugia, Italy \\ ${ }^{10}$ Alexandria University, Alexandria, Egypt \\ * These authors have contributed equally to this work \\ Correspondence to: Lorenzo Leoncini, email: leoncinil@unisi.it
}

Pier Paolo Piccaluga, email: pierpaolo.piccaluga@unibo.it

Keywords: plasmablastic lymphoma; miRNA expression profiling; Burkitt lymphoma; extramedullary plasmacytoma; Epstein-Barr virus; Pathology section

Received: June 20,2017 Accepted: September 08, $2017 \quad$ Published: October 31, 2017

Copyright: Ambrosio et al. This is an open-access article distributed under the terms of the Creative Commons Attribution License 3.0 (CC BY 3.0), which permits unrestricted use, distribution, and reproduction in any medium, provided the original author and source are credited.

\section{ABSTRACT}

Plasmablastic lymphoma (PBL) is an aggressive lymphoma, often arising in the context of immunodeficiency and associated with Epstein-Barr virus (EBV) infection. The most frequently detected genetic alteration is the deregulation of MYC gene through the translocation - $t(8 ; 14)(q 24 ; q 32)$. The diagnosis of PBL is often challenging because it has an overlap in morphology, immunophenotype, cytogenetics and virus association with other lymphomas and plasma cell neoplasms; further, its molecular basis remains elusive. In the present study we aimed to better define the possible contribution of EBV infection as well as miRNA deregulation in PBL pathogenesis.

We studied 23 cases of PBL, 19 Burkitt lymphomas (BL), and 17 extra-medullary plasmacytoma (EMPC). We used qPCR and immunohistochemistry to assess EBV latency patterns, while micro-RNA (miRNA) profiling was performed by next generation sequencing (Illumina) and validated by $\mathrm{qPCR}$.

Our analysis revealed a non-canonical EBV latency program with the partial expression of some proteins characterizing latency II and the activation of an abortive lytic cycle. Moreover, we identified miRNA signatures discriminating PBL from BL and EMPC. Interestingly, based on the miRNA profile, PBL appeared constituted by two discrete subgroups more similar to either BL or EMPC, respectively. This pattern was confirmed in an independent set of cases studied by QPCR and corresponded to different clinico-pathological features in the two groups, including HIV infection, MYC rearrangement and disease localization. 


\section{In conclusion, we uncovered for the first time 1) an atypical EBV latency program in PBL; 2) a miRNA signature distinguishing PBL from the closest malignant counterparts; 3) the molecular basis of PBL heterogeneity.}

\section{INTRODUCTION}

Plasmablastic lymphoma (PBL) is a rare highly aggressive lymphoma that occurs most often in the context of Human Immunodeficiency Virus (HIV)infection $(40-50 \%)$, or in other settings of decreased immune surveillance [1]. Tumor cells have a welldefined morphology resembling B-immunoblasts, and express plasma cells markers [1]. However, a more or less pronounced plasmacytic differentiation as well as a variable expression of CD20 and PAX5 can be encountered [2]. Therefore, the diagnosis of PBL is challenging because it has an overlap in cytomorphologic and immunophenotypic features with plasma cell neoplasms and with lymphomas that show blastic appearance (i.e. more immature, plasmablastlike morphology) [3]. Even using a comprehensive panel of immunostains, a confident differential diagnosis between PBL and anaplastic or plasmablastic extramedullary plasmacytoma (EMPC) is not always possible. Nonetheless, a proper distinction of these entities is mandatory as EMPC and PBL have different prognoses and currently benefit from different therapeutic strategies. On the other hand, the differential diagnosis between PBL and other lymphoma types with blastic appearance is often straightforward due to the lack of B-cell marker expression in PBL. However, BL shares numerous biologic similarities with PBL. In fact, the most frequently detected genomic alteration in PBL is $\mathrm{t}(8 ; 14)$ (q24;q32) leading to $M Y C / I G H$ rearrangement observed in about $50 \%$ of cases $[1,3]$. Other overlaps include cell morphology with the starry sky appearance, the high proliferation rate, the MYC protein over-expression and the association with EBV infection.

A common feature is the presence of the EpsteinBarr virus (EBV) DNA in tumor cells in 50 to $75 \%$ of cases, with higher frequency in HIV-positive cases [1, 3]. The molecular and genetic mechanisms underlying PBL pathogenesis remain largely unknown and are likely dependent on the complexity of biological interplays between immunodeficiency, molecular events (MYC gene rearrangements), co-infecting oncogenic viruses (EBV) [4] and chronic immune activation (expression of immune-checkpoint proteins) [5]. It is unknown, however, whether $M Y C$ translocation represents the initiating or a late genetic event in PBL pathogenesis, and furthermore the latency program of EBV in neoplastic cells is still debated. In fact, although the type I latency is commonly detected, type III latency can be observed in patients with HIV infection and in those patients with post-transplant PBL [3].
In the present study we aimed to better define the pathogenesis and molecular features of PBL by 1) assessing the EBV latency patterns in PBL by qPCR and immunohistochemistry; 2) performing, for the first time to the best of our knowledge, an unbiased miRNA profiling of PBL, EMPC, and BL by next generation sequencing (NGS).

\section{RESULTS}

qPCR and immunohistochemistry reveal a noncanonical latency type and an abortive lytic cycle of EBV in plasmablastic lymphoma

We checked the expression of EBNA-1, EBNA-2, EBNA-3, LMP-1, LMP-2A, EBER transcripts, BZLF-1/ ZEBRA, BMRF-1/Ea-D, BHRF-1/Ea-R, BLLF1/gp350 across the 13 PBL cases (12 EBV-positive and $1 \mathrm{EBV}$ negative). EBNA-1 was detected in $12 / 13$ cases $(92.3 \%)$, LMP-1 and LMP-2 in 1/13 and 10/13 (7.7\% and 76.9\%, respectively) (Figure 1), EBNA-2 and EBNA-3 in 0/13, and EBER in 12/13 (92.3\%). BZLF-1/ZEBRA was found in $12 / 13$ cases $(92.3 \%)$ and both $B M R F-1 /$ Ea-D e BHRF$1 /$ Ea-R in $10 / 13(76.9 \%)$ (Figure 1), while none of the cases expressed BLLF1/gp350 (Figure 1).

We then determined the expression of all the corresponding encoded-proteins. Immunohistochemistry (IHC) confirmed the non-canonical latency associated program with the partial expression of some proteins characterizing latency II (i.e. LMP-1 in 1/13 and LMP2 in $6 / 13$ of the cases) (Figure 1). There was also the expression of proteins characterizing the lytic phase of the virus (i.e. BZLF-1/ZEBRA in 12/13, and BHRF-1/ Ea-R and BMRF-1/Ea-D in 9/13 of the cases) (Figure 1). BLLF1/gp350 was not detected in any case. The qPCR data substantially corresponded to those obtained by IHC; however, slight differences emerged likely due to the higher sensitivity of qPCR.

The same analyses were then carried out in the validation cohort with substantially identical results (Figure 1; Table 3).

Together, these data indicate a non-canonical EBVlatency pattern in PBL. 


\section{Plasmablastic lymphomas can be divided into two groups based on the miRNA profiling}

First, we sought to investigate the relation of PBL with BL and EMPC as far as miRNA expression was concerned. When an unsupervised approach was used, namely PCA, PBL appeared relatively heterogeneous and was not clearly distinct from BL and EMPC, indicating some degree of similarity with both of them. To better determine the possible relation with these two tumors, we developed a miRNA expression-based cell type classifier to establish whether single PBL cases could be related to either one (or none) of the two (BL or EMPC). We found that 7 out of 13 cases were significantly closer to EMPC, 5 out of 13 to BL, and 1 was not clearly classified (Figure 2A-B, Table 4). By a supervised analysis, we identified 136 cellular miRNA differentially expressed in the two new subgroups (Figure 2C; Table 5a and 5b): 123 were up-regulated in "EMPC-related" PBLs, while 13 were up-regulated in "BL-related" ones. Among others, the expression level of hsa-mir-192-5p, hsa-mir-1304-3p, hsamir-148a-3p, hsa-miR-365-3p was studied by RT-qPCR as technical validation. We observed that these miRNA were up-regulated in the cases belonging to the EMPC-related cluster and down-regulated in the BL-related samples, confirming NGS results (Figure 2D). Interestingly, the two groups of PBLs tended to differ also for some clinical features. The patients BL-related were younger (mean age: 50.2 years), with a slight male predominance ( $\mathrm{M}: \mathrm{F}=3: 2)$, an extra-oral localization and a blastic appearance; the majority of them (4 out 5) were HIV-negative. The patients
EMPC-related were older (mean age: 56.85 years), mainly male (M:F=6:1), with an oral localization (4 out 7) and a more pronounced plasmacytic differentiation; the majority of them (4 out 7) were HIV-positive. Of note, 5/5 BLrelated cases presented with $M Y C$ rearrangements at FISH, while only $2 / 7$ EMPC presented with such feature (fisher exact test, $\mathrm{p}=0.03$ ).

When we applied the cell classifier based on the expression of hsa-mir-192-5p, hsa-mir-1304-3p, hsamir-148a-3p, hsa-miR-365-3p to an independent set of 10 cases, we found that $3 / 9$ cases were classified as EMPC-related and 6/9 as BL-related, one case not being evaluable for technical reasons (qPCR failure) (Supplementary Figure 1). Noteworthy, training and test set were comparable concerning HIV infection (6/13 vs $2 / 10$, Fisher exact test, $p=0.09$ )

\section{Plasmablastic lymphomas and plasmacytomas have different miRNA profiles}

In order to unveil specific differences between the PBL and EMPC, we compared the two diseases in term of miRNA expression. When unsupervised approach was adopted, both principal component analysis (PCA) and hierarchical clustering (HC) failed to clearly separate the two entities (data not shown). Then, by supervised analysis, we identified 48 differentially expressed miRNA, including $38 \mathrm{EBV}$-encoded and 10 cellular miRNA which could clearly differentiate the two groups when PCA and HC methods were applied (Figure 3A-3B, Supplementary Table 2). Of note, a linear discriminant analysis indicated

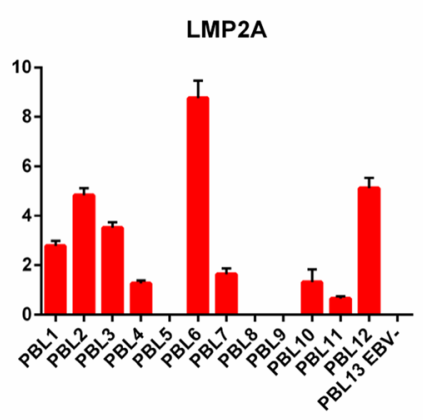

E

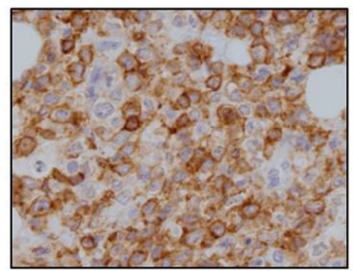

B

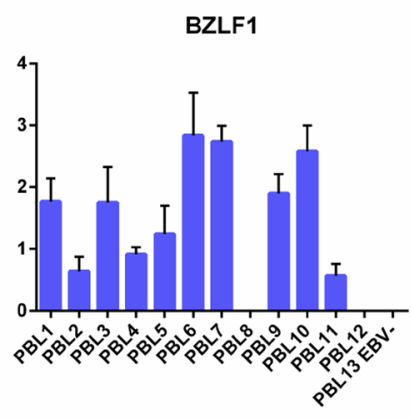

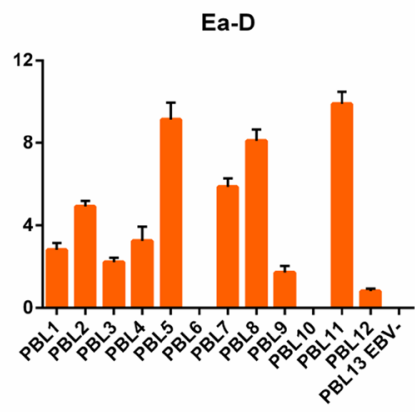

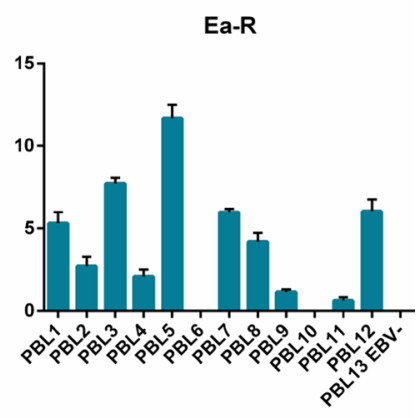

$\mathbf{F}$

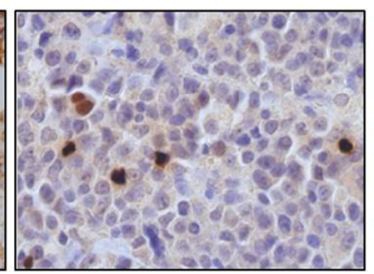

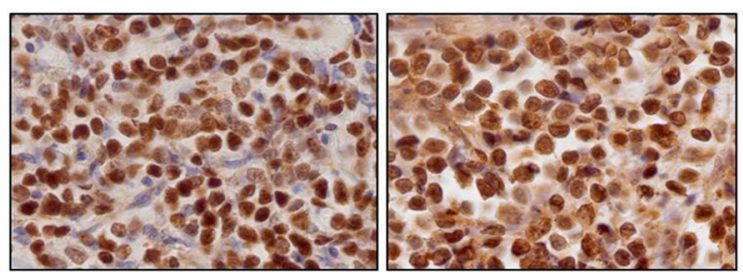

Figure 1: RT-qPCR and immunohistochemistry reveal a non-canonical latency type and an abortive lytic cycle of EBV in neoplastic cells. $L M P-2 A$ A., E., BZLF-1/ZEBRA B., F., BMRF-1/Ea-D C., G., BHRF-1/Ea-R D., H., expression by RT-qPCR (across the 13 PBL cases) and immunohistochemistry (in one exemplificative PBL case; original magnification, 40x). In qPCR graphs, y-axis represents gene expression values (2 Delta $\mathrm{Ct}$ formula). 
Table 1: Clinical, histologic, immunophenotypic and FISH features of discovery cohort patients

\begin{tabular}{|c|c|c|c|c|c|c|}
\hline & \multicolumn{3}{|c|}{ Discovery cohort } & \multicolumn{3}{|c|}{ Validation cohort } \\
\hline & PBL & EMPC & BL & PBL & EMPC & BL \\
\hline $\begin{array}{l}\text { Mean age (range), } \\
\text { years }\end{array}$ & $54.2(28-76)$ & $65.2(44-76)$ & $17.7(3-40)$ & $66.3(45-87)$ & $59(45-74)$ & $18.3(5-43)$ \\
\hline Male:female ratio & $10 / 3$ & $5 / 2$ & $4 / 5$ & $7 / 3$ & $5 / 2(3 \mathrm{NA})$ & $3 / 6$ \\
\hline Oral & 5 & 1 & 1 & 1 & 1 & 1 \\
\hline Extra-Oral & $\begin{array}{l}8 \text { (vulva, ileum, } \\
\text { pleura, penis, skin) }\end{array}$ & $\begin{array}{l}6 \text { (stomach, pleura, } \\
\text { chest-wall, scrotum, } \\
\text { vertebra) }\end{array}$ & $\begin{array}{l}8 \quad \text { (lymph-nodes, } \\
\text { stomach, ileum, } \\
\text { uterus, ovary) }\end{array}$ & $\begin{array}{l}9 \text { (Ileum, stomach, } \\
\text { thyroid, chest-wall, } \\
\text { lymph-node, colon) }\end{array}$ & $\begin{array}{l}9 \quad \text { (iliac bone, } \\
\text { chest-wall, } \\
\text { lymph-node, } \\
\text { vertebra) }\end{array}$ & $\begin{array}{l}8 \quad \text { (ileum, } \\
\text { stomach, lymph- } \\
\text { nodes) }\end{array}$ \\
\hline Morphology & \begin{tabular}{l} 
blastic versus \\
more pronounced \\
plasmacytic \\
differentiation; \\
necrosis, mitotic \\
figures and \\
apoptotic bodies \\
with a "starry-sky" \\
\multicolumn{2}{c}{ appearance }
\end{tabular} & $\begin{array}{l}\text { proliferation of } \\
\text { mature plasma cells; } \\
3 \text { cases more blastic } \\
\text { appearance, 1 case } \\
\text { anaplastic }\end{array}$ & $\begin{array}{l}\text { medium-sized cells, } \\
\text { with monotonous and } \\
\text { uniform appearance, } \\
\text { squared-off } \\
\text { cytoplasm, cohesive } \\
\text { growth and starry-sky } \\
\text { appearance }\end{array}$ & $\begin{array}{l}\text { blastic versus } \\
\text { more pronounced } \\
\text { plasmacytic } \\
\text { differentiation; } \\
\text { necrosis, mitotic } \\
\text { figures and apoptotic } \\
\text { bodies with a "starry- } \\
\text { sky" appearance }\end{array}$ & $\begin{array}{l}\text { proliferation of } \\
\text { mature plasma } \\
\text { cells; } 3 \text { cases } \\
\text { more blastic } \\
\text { appearance, } 1 \\
\text { case anaplastic }\end{array}$ & $\begin{array}{l}\text { medium-sized } \\
\text { cells, with } \\
\text { monotonous } \\
\text { and uniform } \\
\text { appearance, } \\
\text { squared-off } \\
\text { cytoplasm, } \\
\text { cohesive growth } \\
\text { and starry-sky } \\
\text { appearance }\end{array}$ \\
\hline \multicolumn{7}{|l|}{ Immunophenotype } \\
\hline CD38 & 13 & 9 & 9 & 10 & 10 & 9 \\
\hline CD138 & 13 & 9 & 0 & 10 & 10 & - \\
\hline IRF4/MUM1 & 13 & - & 0 & 10 & - & - \\
\hline BCL2 & 13 (weak) & - & 0 & 10 (weak) & - & 0 \\
\hline BCL6 & 13 (weak) & 0 & 9 & 10 (weak) & - & 9 \\
\hline CD10 & 13 (weak) & 0 & 9 & 10 (weak) & - & 9 \\
\hline $\mathrm{CD} 20$ & 0 & 0 & 9 & 0 & 0 & 9 \\
\hline PAX5 & 0 & 0 & 9 & 0 & 0 & - \\
\hline HHV8 & 0 & - & - & 2 & - & - \\
\hline ALK1 & 0 & - & - & 0 & - & - \\
\hline CD56 & 1 & - & - & - & - & - \\
\hline MYC (20-50\%) & 0 & 2 & 0 & 0 & - & 0 \\
\hline MYC (>50\%) & 5 & 0 & 0 & 7 & - & 0 \\
\hline MYC (>80\%) & 8 & 0 & 9 & 2 & - & 9 \\
\hline $\begin{array}{l}\text { MYC translocation } \\
\text { (FISH) }\end{array}$ & $8 / 13$ & $2 / 7$ & $9 / 9$ & $9 / 10$ & $2 / 10$ & $9 / 9$ \\
\hline EBV infection & $12 / 13$ & $2 / 7$ & $5 / 9$ & $4 / 10$ & $0 / 10$ & $6 / 9$ \\
\hline $\begin{array}{l}\text { Immune } \\
\text { suppression }\end{array}$ & $7 / 13$ & NA & NA & $2 / 10$ & NA & NA \\
\hline
\end{tabular}

PBL: plasmablastic lymphoma; EMPC: extramedullary plasmacitoma; BL: Burkitt lymphoma; IHC: immunohistochemistry; pos.: positivity; neg.: negativity; NA.: not available. 
that as few as four miRNA (miR-665, miR-3613-5p, miR1304-3p and miR-3609) could correctly discriminate the samples. Accordingly, based on the expression of such 4 miRNA, we generated a cell classifier and applied it to our series, being able to correctly classify all cases (overall accuracy 100\%; Table 6; Figure 3C). The expression of these four miRNA was also validated by RT-qPCR in the same panel of cases, confirming NGS results (Supplementary Figure 2). Furthermore, for the potential diagnostic implication, we studied their expression by qPCR in an independent validation cohort, consisting of 20 cases (10 PBL and 10 EMPC). When the cell classifier was applied, 9/10 EMPC and 10/10 PBL were correctly identified (95\% overall accuracy; Supplementary Figure 2; Table 7). All the three anaplastic EMPC included in the analysis were correctly classified.

\section{Plasmablastic lymphomas and Burkitt lymphomas have different miRNA profiles}

We then compared PBL to BL. By PCA the two diseases appeared relatively similar, without clear distinction (Figure 4A). By supervised analysis, we could identify a series of 42 differentially expressed miRNA (23 cellular and 19 EBV miRNA) (Figure 4B, Supplementary

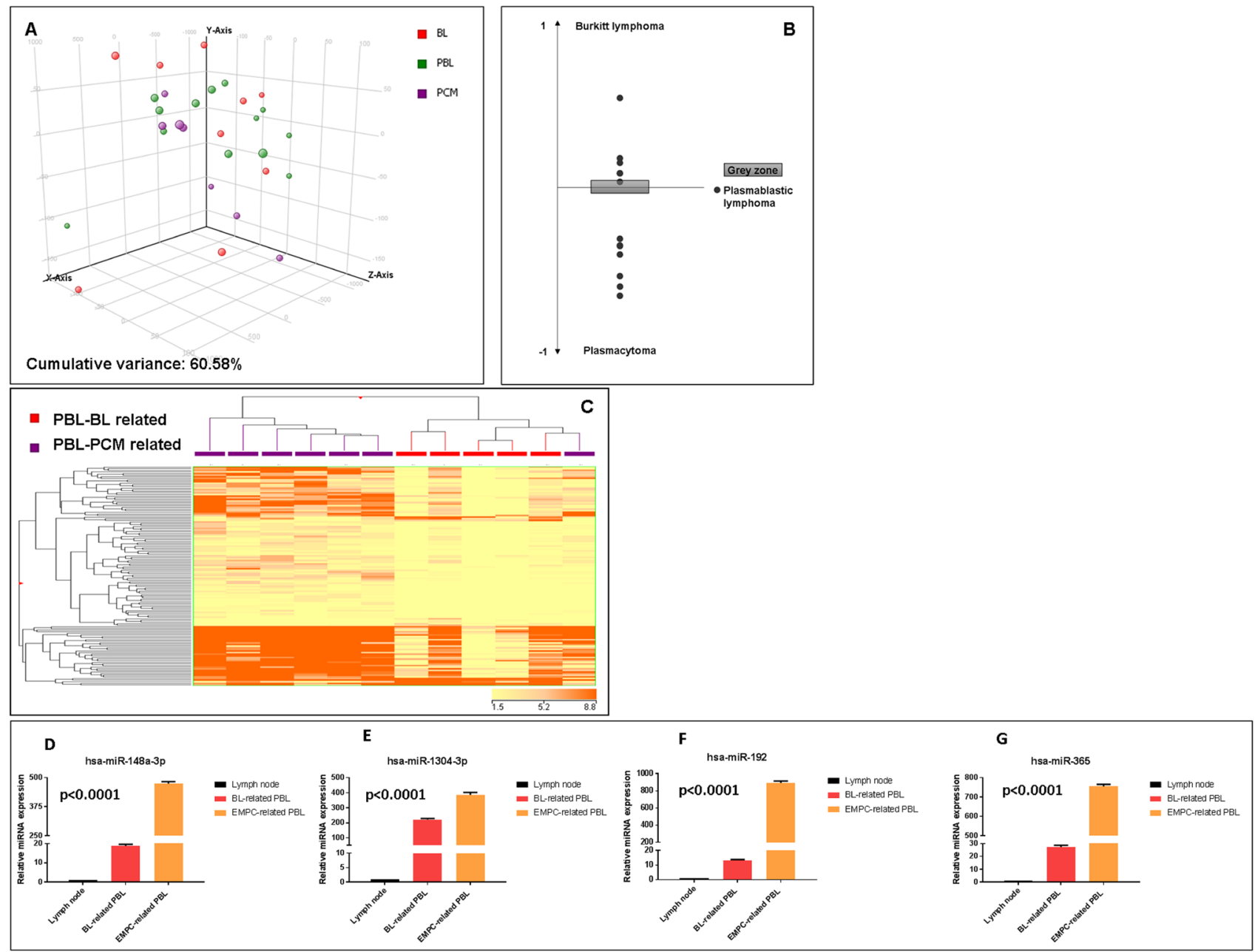

Figure 2: Plasmablastic lymphomas include two molecular subgroups related to either EMPC or BL. Principal component analysis of plasmablastic lymphoma (PBL), extramedullary plasmacytoma (EMPC) and Burkitt lymphoma (BL) cases indicated a basic relation between the three entities at miRNA level A.. A cell type classifier based on a support vector machine algorithm was generated to discriminate BL and EMPC based on the expression of differentially expressed miRNA. When the algorithm was applied to PBL, they were classified as either BL or EMPC indicating their heterogeneity reflecting the similarity to either one of the other two tumor types (BL and EMPC) B.. Grey zone represent value below significance (0.05). Supervised analysis ( $t$-test) comparing BL-related versus EMPC-related PBLs identified a series of differentially expressed genes C.. In the matrix, the dendrogram was generated using a hierarchical clustering algorithm based on the average-linkage method. In the matrix, each column represents a sample and each row represents a gene. The colour scale bar shows the relative gene expression changes normalized by the standard deviation ( 0 is the mean expression level of a given gene). Differential expression of hsa-miR-192-5p, hsa-miR-1304-3p, hsa-miR-148a-3p and hsa-miR-365-3p in BL-related versus EMPC-related PBLs group D.-G. Values are expressed as mean \pm standard deviation (ST). Statistical significance was determined with Student's unpaired $t$-test $(p<0.005)$. 
Table 2: Primers' sets used for qPCR analysis of EBV-encoded genes

\begin{tabular}{|c|c|}
\hline Assay & Primers set \\
\hline \multirow{2}{*}{$E B N A 1$} & 5'- TAC AGG ACC TGG AAA TGG CC- 3' \\
\hline & 5'- TCT TTG AGG TCC ACT GCC G-3' \\
\hline \multirow{2}{*}{$E B N A 2$} & 5'-TAA CCA CCC AGC GCC AAT C-3' \\
\hline & 5'- GTA GGC ATG ATG GCG GCA G 5' \\
\hline \multirow{2}{*}{$E B N A 3 c$} & 5'- CTG GCA AAA CTT GCT CCA- 3' \\
\hline & 5'- GTG CTT CTG CCT TAT CAG A- 3' \\
\hline \multirow{2}{*}{ BHRF1 } & 5'-AGA AAC ACC TCT CCG CCT TT-3' \\
\hline & 5'- ATC CAC ATG TTC GGT GTG TG-3' \\
\hline \multirow{2}{*}{$L M P 1$} & 5'- CAG TCA GGC AAG CCT ATG A-3' \\
\hline & 5'- CTG GTT CCG GTG GAG ATG A-3' \\
\hline \multirow{2}{*}{$B M R F 1$} & 5'- CAACACCGCACTGGAGAG- 3' \\
\hline & 5'- GCCTGCTTCACTTTCTTGG- 3' \\
\hline \multirow{2}{*}{$L M P 2 A$} & 5'- AGC TGT AAC TGT GGT TTC CAT GAC-3' \\
\hline & 5'- GCC CCC TGG CGA AGA G-3' \\
\hline \multirow{2}{*}{$B L L F 1$} & 5'-ATCCAGTTGTATTCAAGGTAGG-3' \\
\hline & 5'-ACTCATTATCACACGAACGG-3' \\
\hline \multirow{2}{*}{$B Z L F 1$} & 5'- AAA TTT AAG AGA TCC TCG TGT AAAACA TC-3' \\
\hline & 5'- CGC CTC CTG TTG AAG CAG AT-3' \\
\hline \multirow{2}{*}{$H P R T$} & 5'-AGC CAG ACT TTG TTG GAT TTG-3' \\
\hline & 5'-TTT ACT GGC GAT GTC AAT AAG-3' \\
\hline
\end{tabular}

Table 3: qPCR and immunohistochemical latency results on validation cohort

\begin{tabular}{|c|c|c|c|c|}
\hline & \multicolumn{2}{|c|}{ RT-qPCR } & \multicolumn{2}{|c|}{ IHC/ISH } \\
\hline & Positive cases (n.) & Negative cases (n.) & Positive cases (n.) & Negative cases (n.) \\
\hline EBNA-1 & 4 & 6 & n.p. & n.p. \\
\hline EBNA-2/3 & 0 & 10 & n.p. & n.p. \\
\hline LMP-1 & 0 & 10 & 0 & 10 \\
\hline LMP-2 & 4 & 6 & 3 & 7 \\
\hline EBER & - & - & 4 & 6 \\
\hline ZEBRA & 3 & 7 & 4 & 6 \\
\hline EaR & 4 & 6 & 3 & 7 \\
\hline EaD & 4 & 6 & 3 & 7 \\
\hline gp350 & 0 & 10 & 0 & 10 \\
\hline
\end{tabular}

ISH: in situ hybridization; n.: number of cases; n.p.: not performed 
Table 3). As technical validation, some of them (hsa-miR148-5p, hsa-miR-1273f, hsa-miR-4488, hsa-miR-494-3p; EBV-miR-BART10-3p, EBV-miR-BART18-3p and EBVmiR-BART19-5p) were also evaluated by qPCR, that again confirmed NGS data (Figure 4C). With the exception of miR-148a-5p, all cellular miRNA were up-regulated in PBL cases in respect to BL samples; a higher expression of all viral miRNA was detected in BL in comparison to PBL group.

These findings were also confirmed in our validation cohort (Supplementary Figure 3).

\section{Plasmablastic lymphomas differ in the miRNA profile based on HIV infection}

Since the association with HIV might affect PBL miRNA profiling, we directly compared HIV-positive versus HIV-negative cases. Unsupervised evaluation by PCA was already able to distinguish the two groups (Figure 5A). Further, the supervised analysis identified as many as 68 differentially expressed miRNA all of which were of cellular origin and were up-regulated in the HIVpositive group (Supplementary Table 4). By contrast, no EBV-encoded miRNA was differentially expressed. Based on the expression of such 68 miRNA, the cases were clearly distinct at $\mathrm{HC}$ (Figure $5 \mathrm{~B}$ ). None of the so far known HIV encoded miRNA emerged as significantly discriminating the two groups at NGS analysis. Therefore, in order to exclude possible underestimation of HIVencoded miRNA contribution, we used a more sensitive qPCR approach to investigate this issue further. Although at low level, we found that both the tested HIV-encoded miRNA (HIV-miR-N-367 and HIV-miR-TAR-3p) were expressed in HIV cases but not in the negative ones (Figure 5C-5D).
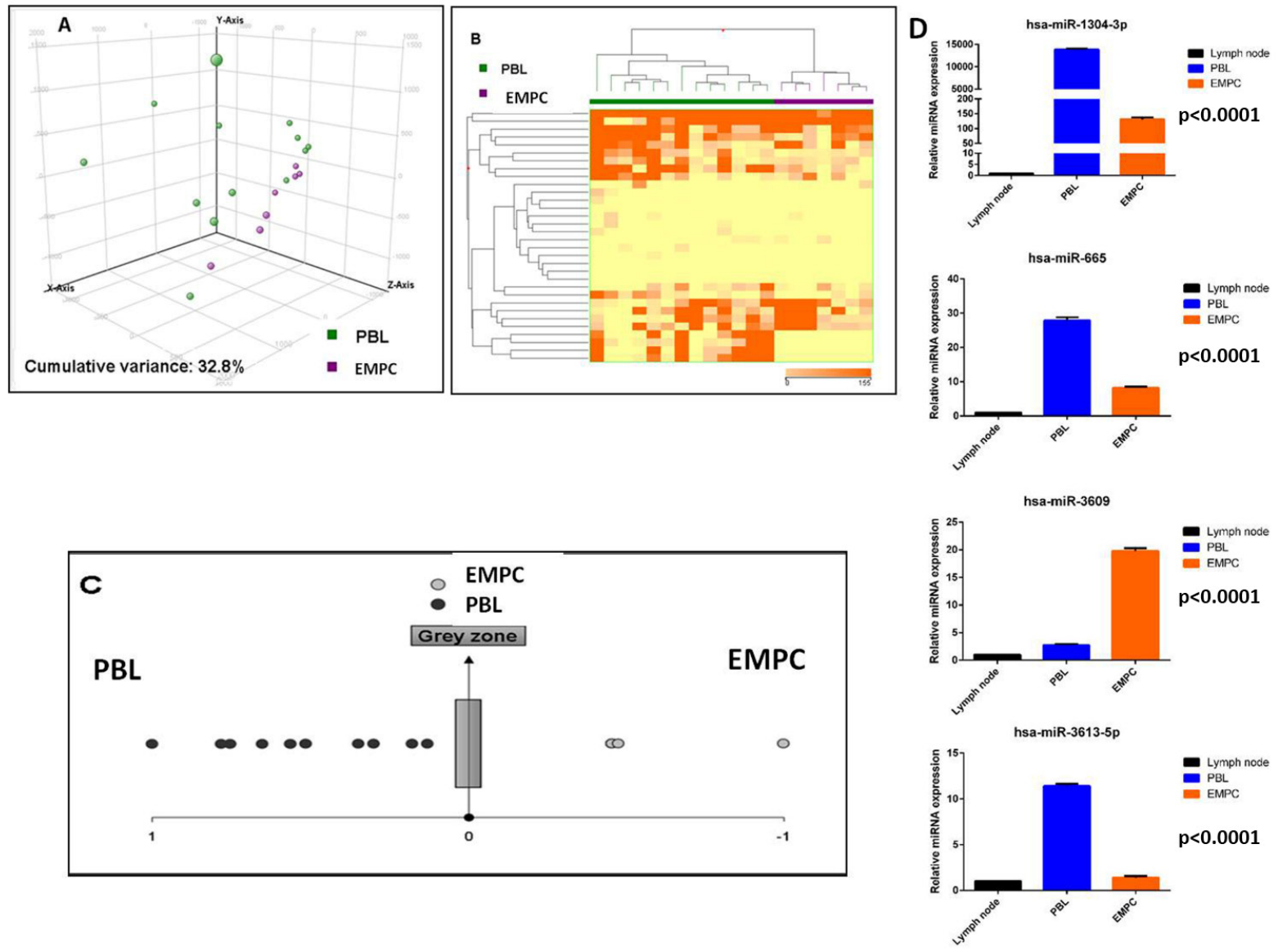

Figure 3: Plasmablastic lymphoma and extramedullary plasmacytoma are distinct based on the miRNA profile. Principal component analysis of plasmablastic lymphoma (PBL) and extramedullary plasmacytoma (EMPC) cases A.. Supervised analysis ( $T$-test) comparing PBL and EMPC cases identified a series of differentially expressed genes B.. In the matrix B, the dendrogram was generated using a hierarchical clustering algorithm based on the average-linkage method. In the matrix, each column represents a sample and each row represents a gene. The colour scale bar shows the relative gene expression changes normalized by the standard deviation $(0$ is the mean expression level of a given gene). A cell type classifier based on a support vector machine algorithm was generated to discriminate PBL and EMPC based on the expression of differentially expressed miRNA C. Grey zone represent value below significance (0.05). Differential expression of hsa-miR-665, hsa-miR-3613-5p, hsa-miR-1304-3p and hsa-miR-3609 in PBL versus EMPC group D.. Values are expressed as mean \pm standard deviation (ST). Statistical significance was determined with Student's unpaired $t$-test $(p<0.005)$. 
Table 4: Correlation between PBL and BL or EMPC

\begin{tabular}{|c|c|c|c|}
\hline Identifier & Cell type & Predicted & Confidence Measure \\
\hline H122 (raw) & PBL & BL & 0.09583959 \\
\hline V109 (raw) & PBL & BL & 0.10874487 \\
\hline s838 (raw) & PBL & BL & 0.1592613 \\
\hline s613 (raw) & PBL & BL & 0.20450206 \\
\hline s806 (raw) & PBL & BL & 0.23559976 \\
\hline s1805 (raw) & PBL & EMPC & 0.27476195 \\
\hline H276 (raw) & PBL & EMPC & 0.45076987 \\
\hline s1529 (raw) & PBL & EMPC & 0.4700034 \\
\hline s13725 (raw) & PBL & EMPC & 0.47044697 \\
\hline s11224 (raw) & PBL & EMPC & 0.5045746 \\
\hline s189 (raw) & PBL & BL & 0.59934336 \\
\hline H333 (raw) & PBL & EMPC & 0.6791907 \\
\hline V376 (raw) & PBL & EMPC & 0.95444125 \\
\hline
\end{tabular}

A

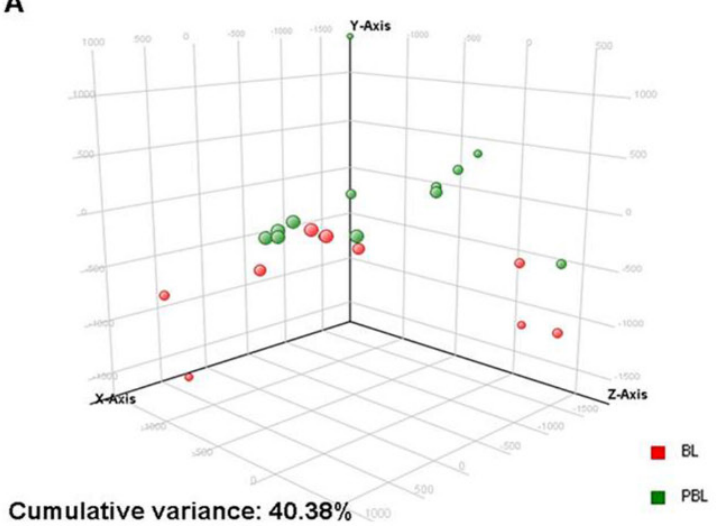

B

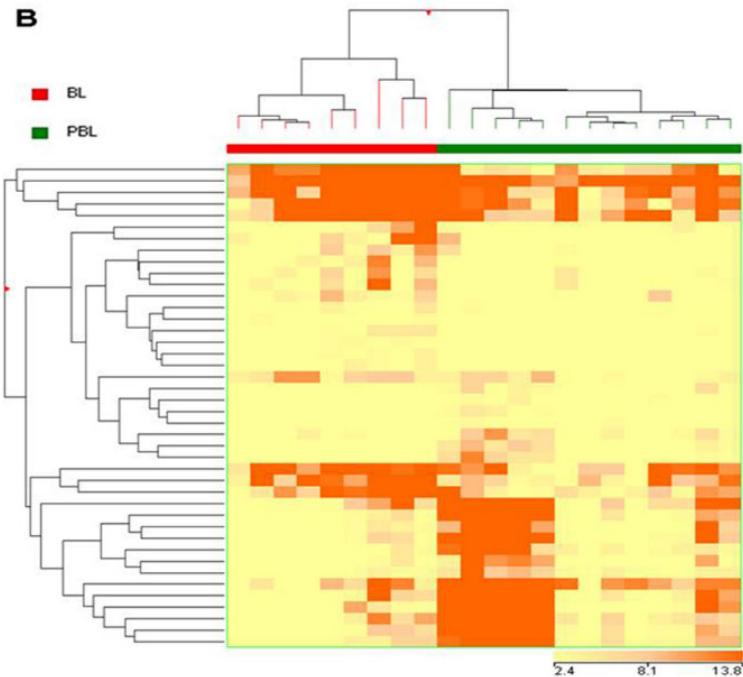

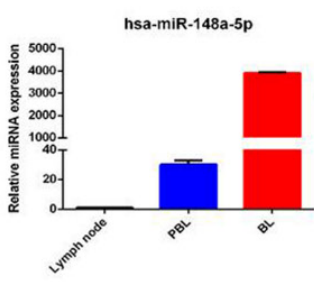
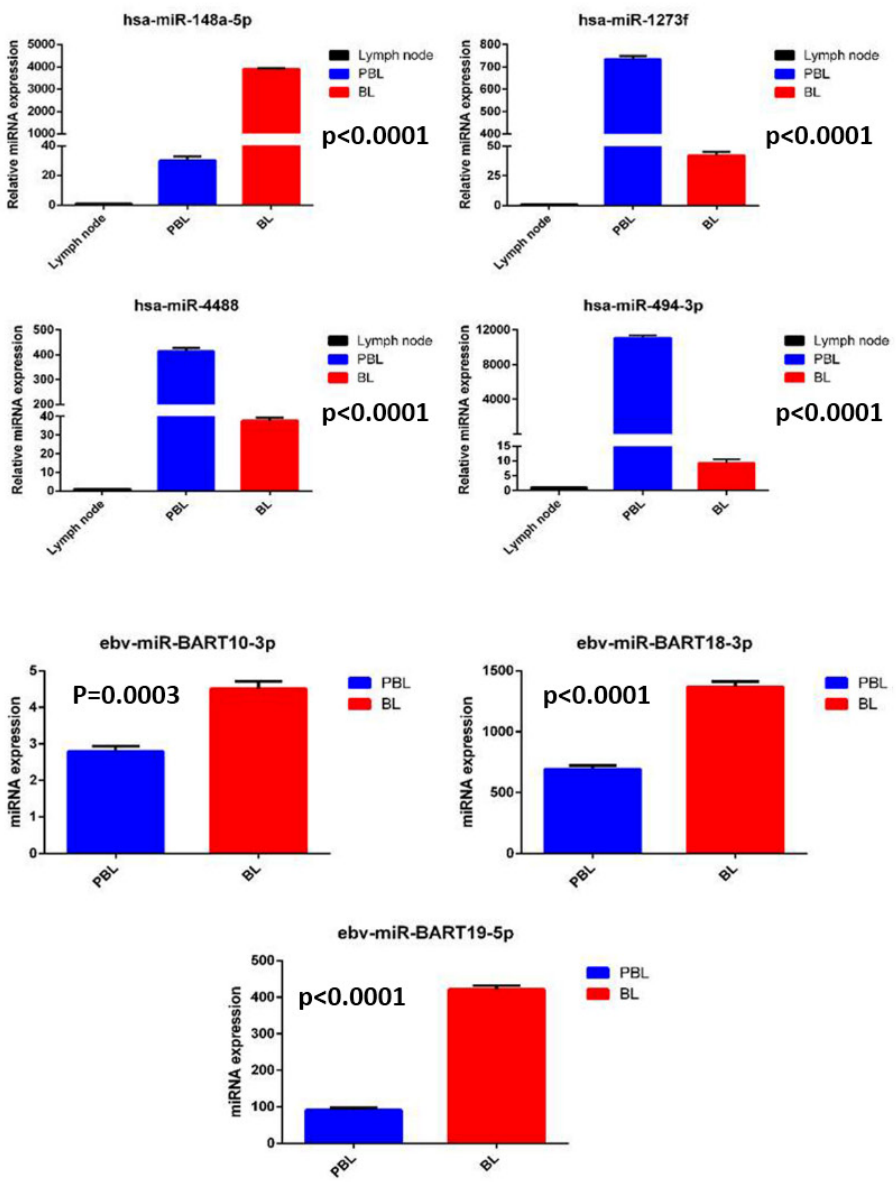

Figure 4: Plasmablastic lymphoma and Burkitt lymphoma are distinct based on the miRNA profile. Principal component analysis of plasmablastic lymphoma (PBL) and Burkitt lymphoma (BL) cases A.. Supervised analysis ( $t$-test) comparing PBL and BL cases identified a series of differentially expressed genes B.. In the matrix B, the dendrogram was generated using a hierarchical clustering algorithm based on the average-linkage method. In the matrix, each column represents a sample and each row represents a gene. The colour scale bar shows the relative gene expression changes normalized by the standard deviation ( 0 is the mean expression level of a given gene). Cellular miRNA (hsa-miR-1273f, hsa-miR-148a-5p, hsa-miR-4488, hsa-miR-494-3p) and EBV-viral miRNA (EBV-miR-Bart10-3p, EBVmiR-18-3p, EBV-miR-19-5p) expression in PBL versus BL cohort groups C. Values are expressed as mean \pm standard deviation (ST). Statistical significance was determined with Student's unpaired $t$-test $(p<0.005)$. 
Table 5A: miRNA differentially expressed in the two PBL groups (BL-related versus EMPC-related)

\begin{tabular}{|c|c|c|c|}
\hline miRNA & p & Fold change & \begin{tabular}{|l} 
Regulation in \\
BL-related \\
group
\end{tabular} \\
\hline hsa-let-7a-5p & 0.028575 & 256 & down \\
\hline hsa-let-7b-5p & 0.006063 & 10809.406 & down \\
\hline hsa-let-7d-3p & 0.044231 & 2.6918004 & down \\
\hline hsa-let-7d-5p & 0.012786 & 3.482202 & down \\
\hline hsa-let-7e-5p & 0.028014 & 4.3297553 & down \\
\hline hsa-let-7f-5p & 0.018527 & 159.15468 & down \\
\hline hsa-let-7g-5p & 0.046021 & 13.387893 & down \\
\hline hsa-miR-101-3p & 0.002134 & 48.502926 & down \\
\hline hsa-miR-103a-3p & 0.004657 & 21.5344 & down \\
\hline hsa-miR-103b & 0.011111 & 18.74678 & down \\
\hline hsa-miR-106b-3p & 0.02385 & 4.594794 & down \\
\hline hsa-miR-106b-5p & 0.025859 & 13.125367 & down \\
\hline hsa-miR-10b-5p & 0.045394 & $1.17 \mathrm{E}+07$ & down \\
\hline hsa-miR-125a-5p & 0.022511 & 40.58451 & down \\
\hline hsa-miR-125b-1-3p & 0.002118 & 2.6918004 & down \\
\hline hsa-miR-126-3p & 0.012516 & 128 & down \\
\hline hsa-miR-126-5p & 0.0226 & 159.15468 & down \\
\hline hsa-miR-127-3p & 0.030994 & 86.13763 & down \\
\hline hsa-miR-128-3p & 0.00253 & 8.659511 & down \\
\hline hsa-miR-1304-3p & 0.039182 & 5.0730634 & down \\
\hline hsa-miR-130a-3p & 0.015367 & 5.0730634 & down \\
\hline hsa-miR-130b-3p & 0.024846 & 5.278032 & down \\
\hline hsa-miR-132-3p & 0.003019 & 3.8446627 & down \\
\hline hsa-miR-139-5p & 0.037432 & 2.122426 & down \\
\hline hsa-miR-140-3p & 0.004751 & 436.9814 & down \\
\hline hsa-miR-140-5p & 0.038232 & 2.164878 & down \\
\hline hsa-miR-143-3p & 0.023024 & 40743.586 & down \\
\hline hsa-miR-145-3p & 0.034978 & 4.780439 & down \\
\hline hsa-miR-145-5p & 0.019368 & 26.250734 & down \\
\hline hsa-miR-146a-5p & 0.002346 & 111.43049 & down \\
\hline hsa-miR-146b-5p & 0.003099 & 1176.267 & down \\
\hline hsa-miR-148a-3p & 0.003072 & $1.38 \mathrm{E}+10$ & down \\
\hline hsa-miR-148b-3p & 0.001742 & 13.387893 & down \\
\hline hsa-miR-151a-3p & 0.017852 & 49.47307 & down \\
\hline hsa-miR-155-5p & 0.042635 & 62.74499 & down \\
\hline hsa-miR-15a-5p & 0.01427 & 4.9735837 & down \\
\hline hsa-miR-15b-5p & 0.029421 & 3.0920668 & down \\
\hline hsa-miR-16-5p & 0.002014 & 5967.2734 & down \\
\hline hsa-miR-181a-3p & 0.00621 & 2.390219 & down \\
\hline hsa-miR-181a-5p & 0.003179 & 26353.643 & down \\
\hline hsa-miR-181b-5p & 0.028867 & 218.4907 & down \\
\hline hsa-miR-181c-5p & 0.024008 & 5.0730634 & down \\
\hline hsa-miR-182-5p & 0.041277 & 141.3235 & down \\
\hline hsa-miR-183-5p & 0.017472 & 34.638054 & down \\
\hline hsa-miR-186-5p & 0.006628 & 182.82076 & down \\
\hline hsa-miR-191-5p & 0.003595 & 6854.601 & down \\
\hline hsa-miR-192-5p & 0.003457 & 10.146127 & down \\
\hline hsa-miR-193b-3p & 0.003606 & 179.23567 & down \\
\hline hsa-miR-195-5p & 0.037465 & 3.3469734 & down \\
\hline
\end{tabular}


Table 5B: miRNA differentially expressed in the two PBL groups (BL-related versus EMPC-related)

\begin{tabular}{|c|c|c|c|}
\hline miRNA & $\mathbf{p}$ & Fold change & \begin{tabular}{|l} 
Regulation in \\
BL-related \\
group
\end{tabular} \\
\hline hsa-miR-196a-5p & 0.033883 & 9.373392 & down \\
\hline hsa-miR-197-3p & 0.02192 & 13.387893 & down \\
\hline hsa-miR-199a-3p & 0.043553 & 8.489703 & down \\
\hline hsa-miR-210-3p & 0.020199 & 5.1745324 & down \\
\hline hsa-miR-2110 & 0.020158 & 2 & down \\
\hline hsa-miR-212-3p & 0.037432 & 2.122426 & down \\
\hline hsa-miR-21-3p & 0.027591 & 25.73597 & down \\
\hline hsa-miR-214-3p & 0.016095 & 5.1745324 & down \\
\hline hsa-miR-21-5p & 0.016598 & $1.60 \mathrm{E}+09$ & down \\
\hline hsa-miR-221-3p & 0.009039 & 194.0117 & down \\
\hline hsa-miR-221-5p & 0.019567 & 3.482202 & down \\
\hline hsa-miR-222-3p & 0.001477 & 120.616684 & down \\
\hline hsa-miR-22-3p & 0.021866 & 144.15012 & down \\
\hline hsa-miR-23a-3p & 0.008328 & 48.502926 & down \\
\hline hsa-miR-23b-3p & 0.03084 & 48.502926 & down \\
\hline hsa-miR-24-3p & 0.047314 & 13.655676 & down \\
\hline hsa-miR-25-3p & 4.42E-04 & 324.6761 & down \\
\hline hsa-miR-26a-5p & 0.006917 & 8693.463 & down \\
\hline hsa-miR-26b-5p & $8.58 \mathrm{E}-04$ & 93.23874 & down \\
\hline hsa-miR-27a-3p & 0.011841 & 1176.267 & down \\
\hline hsa-miR-27b-3p & 0.011343 & 512 & down \\
\hline hsa-miR-27b-5p & 0.048211 & 2 & down \\
\hline hsa-miR-28-3p & 0.001606 & 50.4626 & down \\
\hline hsa-miR-28-5p & 7.68E-04 & 6.0628657 & down \\
\hline hsa-miR-29b-3p & 0.012198 & 5.6011167 & down \\
\hline hsa-miR-29c-3p & 0.004106 & 7.3907156 & down \\
\hline hsa-miR-29c-5p & 0.001043 & 3.482202 & down \\
\hline hsa-miR-301a-3p & 0.00456 & 4.504692 & down \\
\hline hsa-miR-3074-5p & 0.00282 & 5.3836007 & down \\
\hline hsa-miR-30a-5p & 0.028991 & 70.66175 & down \\
\hline hsa-miR-30b-5p & 0.006546 & 7.1037035 & down \\
\hline hsa-miR-30c-5p & 0.003975 & 15.686253 & down \\
\hline hsa-miR-30d-5p & 0.020276 & 86.13763 & down \\
\hline hsa-miR-30e-3p & 0.009217 & 5.6011167 & down \\
\hline hsa-miR-30e-5p & 0.012316 & 231.86519 & down \\
\hline hsa-miR-3184-3p & 0.036321 & 3.8446627 & down \\
\hline hsa-miR-3184-5p & 0.047828 & 29.562862 & down \\
\hline hsa-miR-324-5p & 0.007311 & 2.8565736 & down \\
\hline hsa-miR-326 & 0.017578 & 2.6918004 & down \\
\hline hsa-miR-328-3p & 0.040136 & 3.6228948 & down \\
\hline hsa-miR-340-5p & 0.029992 & 2.6918004 & down \\
\hline hsa-miR-345-5p & $3.51 \mathrm{E}-05$ & 5.3836007 & down \\
\hline hsa-miR-34a-5p & 0.025859 & 13.125367 & down \\
\hline hsa-miR-3613-5p & 0.017127 & 3.2169964 & down \\
\hline hsa-miR-361-3p & 0.018605 & 9.009385 & down \\
\hline hsa-miR-3615 & 0.006715 & 6.562682 & down \\
\hline hsa-miR-361-5p & 0.011483 & 5.7131467 & down \\
\hline hsa-miR-365a-3p & 0.024084 & 2.3433483 & down \\
\hline hsa-miR-374a-5p & 0.001147 & 2.4867918 & down \\
\hline
\end{tabular}




\begin{tabular}{|c|c|c|c|}
\hline hsa-miR-374b-5p & 7.10E-04 & 3.1539128 & down \\
\hline hsa-miR-378a-3p & 0.010879 & 59.125725 & down \\
\hline hsa-miR-378d & 0.007875 & 3.1539128 & down \\
\hline hsa-miR-381-3p & 0.015433 & 2.122426 & down \\
\hline hsa-miR-423-3p & 0.01819 & 64 & down \\
\hline hsa-miR-425-5p & 0.025742 & 10.146127 & down \\
\hline hsa-miR-4448 & 0.043389 & 18.01877 & down \\
\hline hsa-miR-454-3p & 0.008394 & 5.0730634 & down \\
\hline hsa-miR-4633-5p & 0.030738 & 49.47307 & down \\
\hline hsa-miR-497-5p & 0.008057 & 4.244852 & down \\
\hline hsa-miR-500a-3p & 0.01973 & 3.0920668 & down \\
\hline hsa-miR-502-3p & 0.015503 & 3.031433 & down \\
\hline hsa-miR-574-3p & 0.018238 & 3.9215622 & down \\
\hline hsa-miR-615-3p & 0.032965 & 3.1539128 & down \\
\hline hsa-miR-625-3p & 0.016429 & 7.390717 & down \\
\hline hsa-miR-629-5p & 0.012786 & 3.482202 & down \\
\hline hsa-miR-652-3p & 0.037863 & 7.843124 & down \\
\hline hsa-miR-654-3p & 0.042732 & 2.3433483 & down \\
\hline hsa-miR-660-5p & 0.023198 & 2.5365317 & down \\
\hline hsa-miR-664a-3p & 0.007032 & 2.4867918 & down \\
\hline hsa-miR-6737-5p & 0.028424 & 2.0400033 & down \\
\hline hsa-miR-92b-3p & 0.004056 & 1747.9257 & down \\
\hline hsa-miR-93-5p & 0.014503 & 56.829643 & down \\
\hline hsa-miR-96-5p & 0.027386 & 3.482202 & down \\
\hline hsa-miR-98-5p & 0.037122 & 3.4139185 & down \\
\hline hsa-miR-3178 & 0.025964 & 66.58581 & up \\
\hline hsa-miR-3648 & 0.010903 & 4.080006 & up \\
\hline hsa-miR-4492 & 0.037656 & 91768.664 & up \\
\hline hsa-miR-4497 & 0.029851 & 3859.7363 & up \\
\hline hsa-miR-4508 & 0.039168 & 1108.4174 & up \\
\hline hsa-miR-5787 & 0.01597 & 22492.303 & up \\
\hline hsa-miR-6087 & 0.032981 & 14.207409 & up \\
\hline hsa-miR-6126 & 0.001043 & 12.125731 & up \\
\hline hsa-miR-663b & 0.019219 & 5.6011167 & up \\
\hline hsa-miR-6739-5p & $1.33 \mathrm{E}-04$ & 3.482202 & up \\
\hline hsa-miR-6743-5p & 0.029317 & 4.594794 & up \\
\hline hsa-miR-7704 & 0.026789 & 62990.94 & up \\
\hline hsa-miR-8072 & 0.034227 & 2.0808065 & up \\
\hline
\end{tabular}

Key cellular signaling pathways and metabolic activities may be affected by deregulated cellular and viral miRNA

To explore the potential involvement of the detected miRNA in PBL pathogenesis, we searched for genes targeted by the miRNA discriminating the molecular subtypes of PBL that were previously confirmed as direct targets biochemically. Among others, we found genes encoding for tumor suppressors (PTEN, $P B X 2)$ and lipid metabolism controller (PPARGC1A, PLIN2/adipophilin) whose expression levels were found to be regulated by BART-19-5p, and FOXK2, a gene involved in the control of viral and cellular promoter elements, regulated by hsamiR148a-5p, has-miR-3609, has-miR-365.

The expression level of the encoded proteins was then checked by immunohistochemistry in 13 PBL cases. BL-related cases presented with significantly higher degree of expression of PTEN, PBX2, PPARGC1A, PLIN2/adipophilin and FOXK2 (Figure 6A-6E and) $(\mathrm{p}<0.05)$.

We also investigated the potential pathobiological relevance of these genes by gene set enrichment analysis (GSEA). We found several gene sets significantly enriched; among others those associated to Kruppellike factor 1 (KLF1), enhancer of zeste 2 polycomb repressive complex 2 subunit (EZH2) and $M Y C$ appeared 
of particular interest due to the high scores and consistent recurrence (Supplementary Table 5).

\section{Novel miRNA discovery}

We analyzed 13 PBL miRNA-seq libraries to identify candidate novel miRNA species that were deregulated in this tumor type. After sequence filtering, we enumerated 133 candidate novel miRNA, not identified in miRBase v21 (Supplementary Table 6). Differential expression analysis was carried out between PBL versus EMPC and PBL versus BL. We found 27 miRNA differentially expressed between $\mathrm{PBL}$ and $\mathrm{BL}$, and 7 between PBL and EMPC (Supplementary Tables 7-8). Further, 17 miRNA discriminated the HIV positive cases from the HIV negative ones (Supplementary Table 9). Conversely, none of the putative novel miRNA were differentially expressed in PBLs according to their relation with EMPC or BL. Of note, one of these novel putative
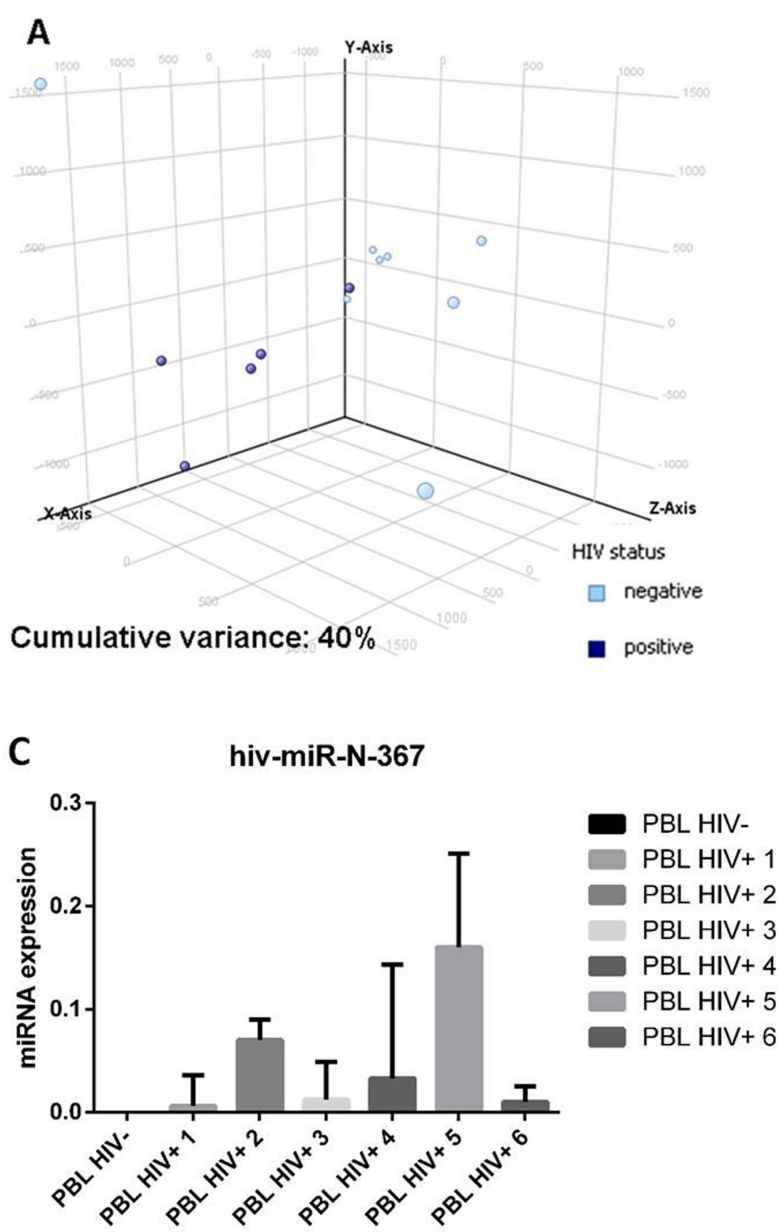

miRNA mapped on the HIV genome. However, these preliminary observations need appropriate validation.

\section{DISCUSSION}

The pathogenesis of PBL has not been clearly defined but most likely it involves deregulation of terminal B-cell differentiation and apoptosis, via the effect of $M Y C$ gene rearrangements and possibly EBV infection [3]. To better understand the viral program expressed in PBLs, we performed a complete assessment of EBV-encoded genes and proteins, demonstrating a non-canonical latency-associated program with the expression of latency II characterizing viral proteins along with sporadic lytic cycle reactivation. Nonetheless, the absence of BLLF1/ gp350 expression in all the cases indicated an abortive lytic cycle in neoplastic cells. These results are consistent with previous findings, suggesting that EBV lytic reactivation may have pathogenic relevance in the context of both
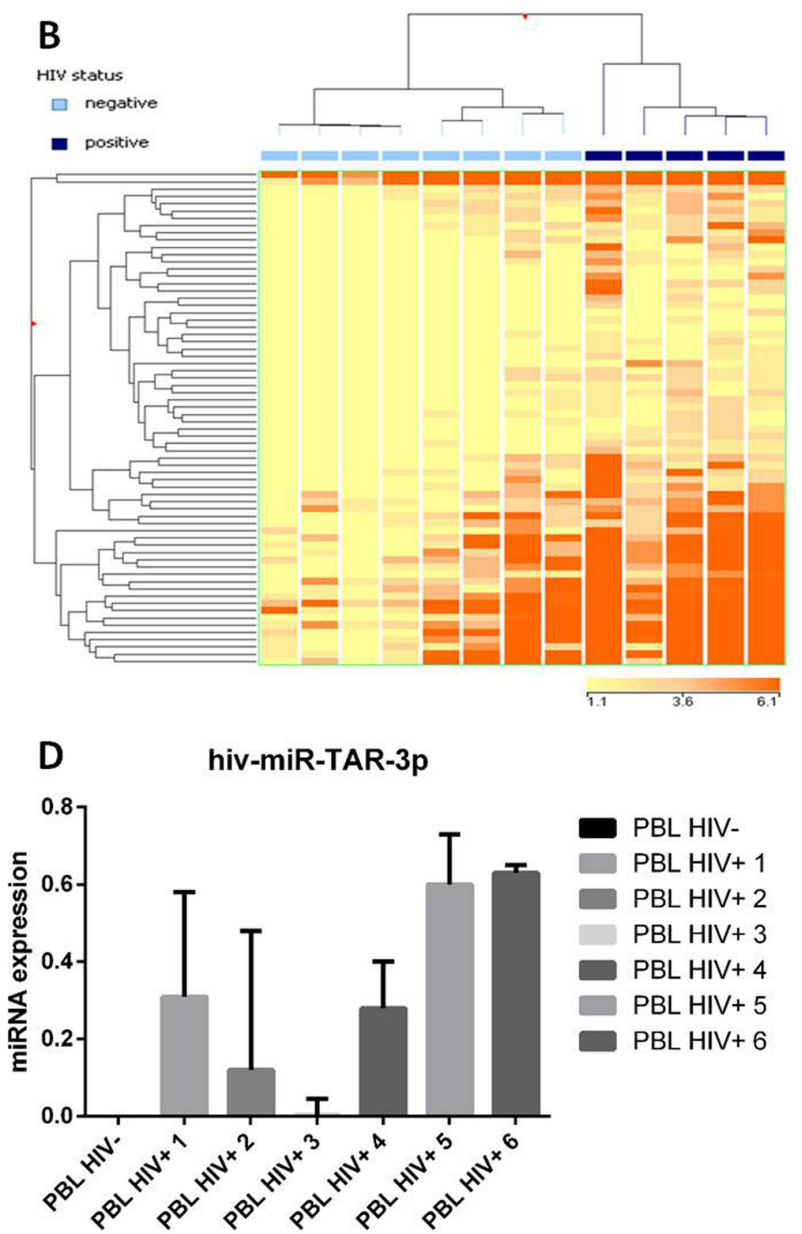

Figure 5: Plasmablastic lymphomas differ in their miRNA profile according to the presence of HIV. Principal component analysis of HIV-positive and HIV-negative plasmablastic lymphomas (PBLs) indicated a rough distinction of the two groups based on miRNA expression A.. Supervised analysis (T-test) comparing HIV-positive versus HIV-negative PBLs identified a series of differentially expressed genes B.. In the matrix, the dendrogram was generated using a hierarchical clustering algorithm based on the average-linkage method. In the matrix, each column represents a sample and each row represents a gene. The colour scale bar shows the relative gene expression changes normalized by the standard deviation ( 0 is the mean expression level of a given gene). 5C. HIV-miR-367 expression in HIV positive and HIV negative PBL cases C.. HIV-miR-TAR-3p expression in HIV positive and HIV negative PBL cases D.. 
Table 6: Molecular diagnosis of PBL versus EMPC: diagnostic accuracy of the 4-miRNA based classifier in the discovery set

\begin{tabular}{|c|c|c|c|}
\hline & & \multicolumn{2}{|c|}{ Conventional histopathology } \\
\hline & & PBL & EMPC \\
\hline \multirow{2}{*}{ miRNA profiling } & $P B L$ & 13 & 0 \\
\hline & $E M P C$ & 0 & 7 \\
\hline & & Value & $95 \%$ CI \\
\hline \multirow{8}{*}{ Accuracy } & SP & $100 \%$ & $100-100$ \\
\hline & ST & $100 \%$ & $100-100$ \\
\hline & PPV & $100 \%$ & $100-100$ \\
\hline & NPV & $100 \%$ & $100-100$ \\
\hline & $\mathbf{L R}+$ & 10 & 1.56 to 64.20 \\
\hline & LR- & $\mathrm{NC}$ & $\mathrm{NC}$ \\
\hline & Overall accuracy & \multicolumn{2}{|c|}{$100 \%(20 / 20)$} \\
\hline & Pre-test probability & $50 \%$ & $28-72$ \\
\hline
\end{tabular}

Table 7: Molecular diagnosis of PBL versus EMPC: diagnostic accuracy of the 4-miRNA based classifier in the validation set

\begin{tabular}{|c|c|c|c|}
\hline & & \multicolumn{2}{|c|}{ Conventional histopathology } \\
\hline & & PBL & EMPC \\
\hline \multirow{2}{*}{ miRNA profiling } & $P B L$ & 10 & 1 \\
\hline & $E M P C$ & 0 & 9 \\
\hline & & Value & $95 \%$ CI \\
\hline \multirow{8}{*}{ Accuracy } & SP & $90 \%$ & $71-100$ \\
\hline & ST & $100 \%$ & $100-100$ \\
\hline & PPV & $91 \%$ & $74-100$ \\
\hline & NPV & $100 \%$ & $100-100$ \\
\hline & LR+ & 10 & 1.56 to 64.20 \\
\hline & LR- & $\mathrm{NC}$ & $\mathrm{NC}$ \\
\hline & Overall accuracy & \multicolumn{2}{|c|}{$95 \%(19 / 20)$} \\
\hline & Pre-test probability & $50 \%$ & $28-72$ \\
\hline
\end{tabular}

active and depressed immune response [6]. Intriguingly, we have recently demonstrated a non-canonical latency program with activation of the lytic cycle also in endemic BL cases, highlighting another overlap between PBL and BL [7].

The limited knowledge on PBL biology likely contributes to suboptimal management and poor prognosis of the patients. Therefore, in the present study we attempted to shed new light on the molecular bases of PBL. We studied for the first time the miRNA expression profile of PBL and compared it with those of BL and EMPC, chosen as the closest neoplastic counterparts considering morphological, immunophenotypic, genetic and epidemiological factors.

For the first time we observed that PBL include at least two molecular subgroups that presented with clinico-pathological peculiar features, including $M Y C$ rearrangements and HIV-association that appeared as main drivers of this sub-classification. On the other hand, this might be also due to a dissimilar histogenesis, genetic 
background and/or microenvironmental influences $[5,7$, 8]. Interestingly, we found that the two PBL subgroups differed for the expression of proteins, the regulation of which might be sustained by the differentially expressed miRNA. These proteins turned out to be involved in relevant cellular processes including cell cycle and proliferation, signal transduction, apoptosis, viral replication, cell metabolism, epigenetic regulation of transcription (via EZH2) and differentiation. Of note, our findings unveiled another overlap between PBL and BL

\section{EMPC-related}

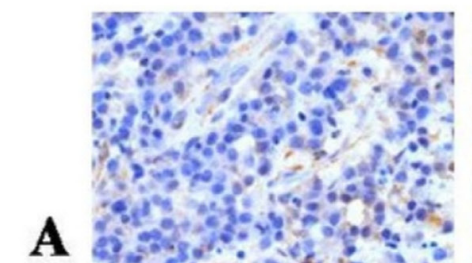

$\mathbf{A}$
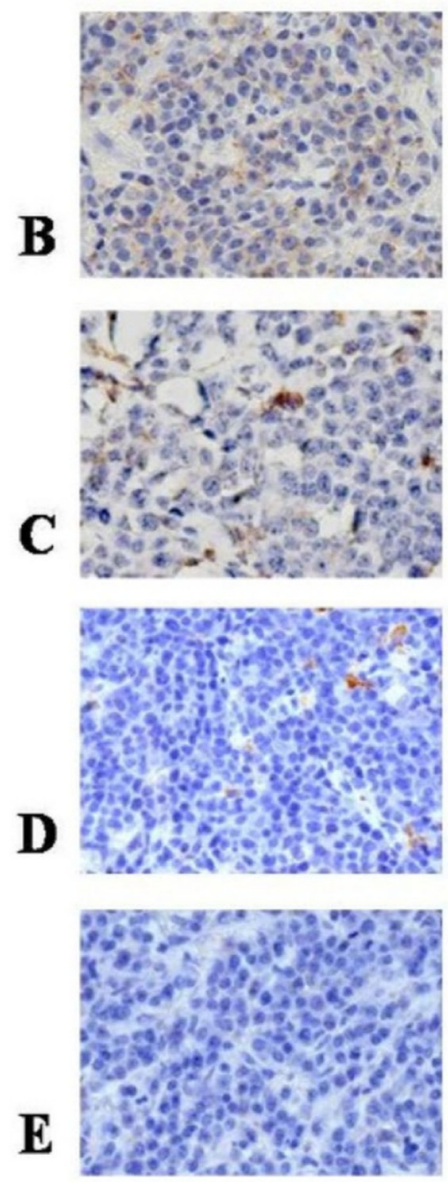

BL-related
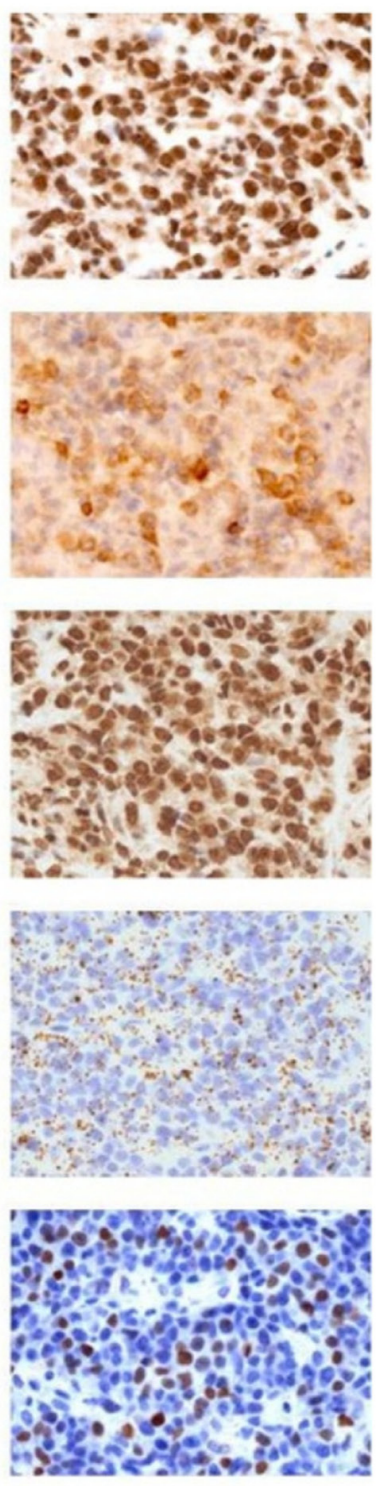

related to the metabolic reprogramming of the neoplastic cells [9]. In fact, both PBL and BL showed an activation of the lipid metabolism characterized by the expression of adipophilin, likely related to MYC activity as previously reported in the B-cell setting $[10,11]$.

Furthermore, we identified specific differences between PBL and both BL and EMPC as far as miRNA expression was concerned. Of note, based on the expression of as few as 4 miRNA, we could efficiently perform a molecular differential diagnosis between

\section{EMPC-related BL-related}
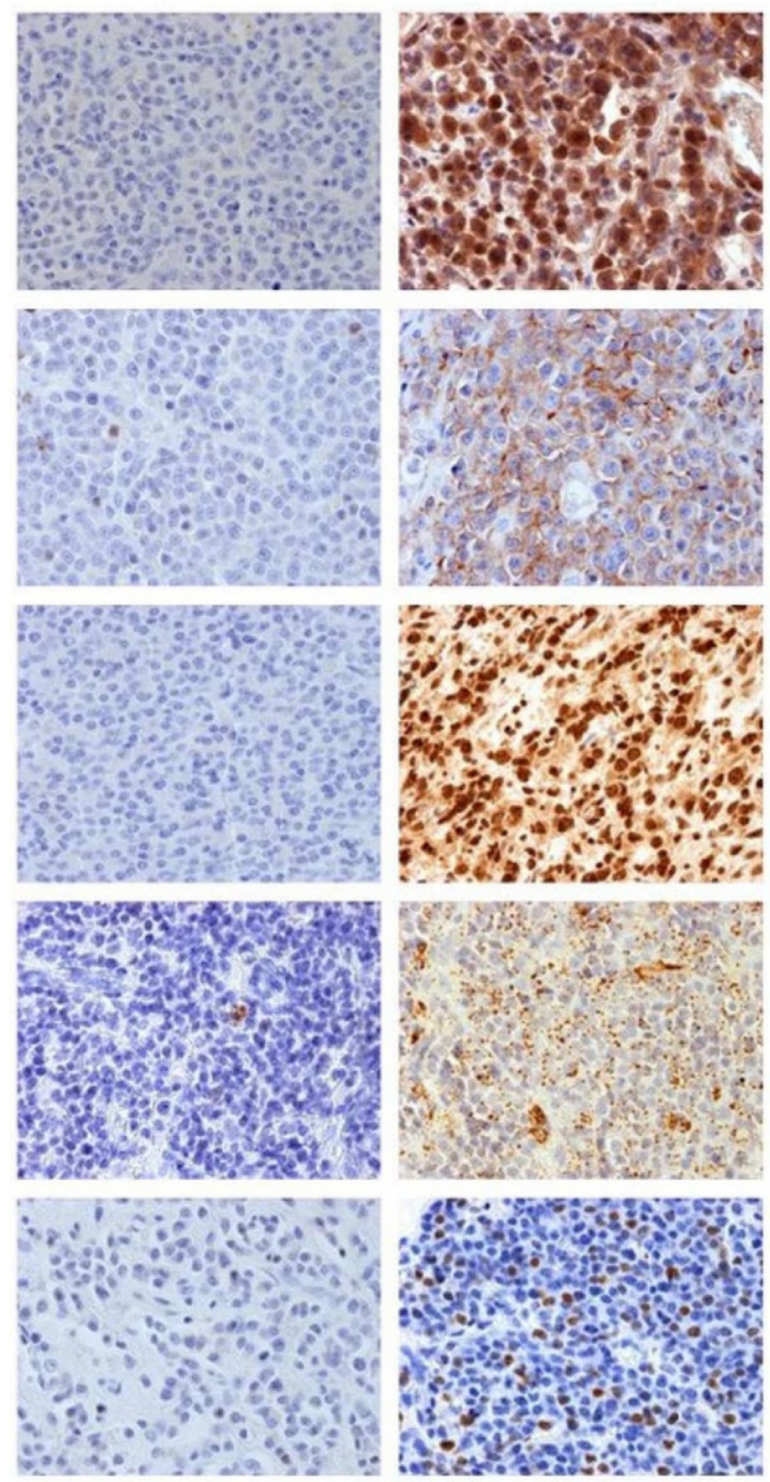

\section{Discovery cohort $(\mathrm{N}=13)$}

\section{Validation cohort $(\mathrm{N}=10)$}

Figure 6: PTEN, PBX2, PPARGC1A, PLIN2/adipophilin, and FOXK2 protein expression in the two sub-groups of PBL. PTEN, PBX2, PPARGC1A, PLIN2/adipophilin and FOXK2 showed lower level of expression in all the PBL cases that clustered closer to the EMPC cases than to the BL cases; one representative case for each sub-group is depicted. A.-E., O.M.: 40x. PBL: plasmablastic lymphoma; EMPC: extramedullary plasmacytoma; BL: Burkitt lymphoma. 
PBL and EMPC, even when anaplastic/plasmablastic EMPC were included. The latter, at present constitute real diagnostic challenges even for most experienced hematopathologists. Here, we demonstrated the efficiency of the molecular diagnostic tool in two independent cohorts of unselected cases, fulfilling the STARD criteria for molecular diagnostic. Phase 4 diagnostic accuracy studies are now warranted to prospectively evaluate the clinical utility of the test.

Finally, we observed that PBL molecular features are at least in part dependent on HIV status. Different mechanisms might be in place how HIV might contribute to $\mathrm{PBL}$ pathogenesis: duration and the degree of immunodeficiency, loss of immune control of oncogenic herpesviruses as EBV, chronic B-cell proliferation/ exhaustion due to chronic antigenic stimulation, and/ or factors unrelated to immune dysfunction, including a theoretically possible direct oncogenic effect. Actually, the involvement of HIV infection in PBL pathogenesis may explain the high frequency of this virus in PBL patients. In our analysis, HIV-positive and HIV-negative PBL cases differed in terms of cellular miRNA. This might be due in part to HIV itself by its own miRNA. However, only when qPCR was performed we could detect very low amounts of HIV-encoded miRNA in PBL cases. This likely reflected the inferior sensitivity of RNAseq analysis, especially when filters improving specificity and robustness are applied to RNAseq analysis. Indeed, the detection of viral miRNA is more likely consistent with the hypothesis that they were encoded in scattered T-cells, macrophages or dendritic cells rather than in the neoplastic clone. Nevertheless, their potential pathogenetic role in PBL cannot be excluded as miRNA are easily transferred through the tissues in the tumor microenvironment. Moreover, we identified by NGS a putative novel miRNA mapping on the HIV genome and highly expressed by PBL cells, the significance of which definitely need further analysis.

The major limitation of the study is probably the relatively low number of PBL cases analyzed $(\mathrm{N}=23)$. Nonetheless, it should be noted that all results have been validated in a cohort of cases independent from the discovery one, this making quite solid our assumptions [12].

In conclusion, the present study offers novel insights on PBL biology and pathogenesis that might be useful for the future management of this orphan disease in terms of classification, diagnosis and treatment. Particularly, an improved understanding of the complex interplay between genetic lesion and viral effects, as well as the multiple mechanisms regulating EBV reactivation might lead to the development of more specific therapies including miRNAtargeted therapies, MYC signaling pathways inhibitors, and combination of EBV lytic phase induction and antiEBV drugs [13].

\section{MATERIALS AND METHODS}

\section{Cases selection, immunohistochemistry, fluorescent in situ hybridization (FISH) and EBER-in-situ hybridization}

For this study 23 PBL, 17 EMPC and 18 BL cases (9 endemic, 9 sporadic), collected at the Departments of Pathology of Aga Khan University Hospital, Nairobi (Kenya), Aga Khan Hospital, Kisumu (Kenya), Terni Hospital (Italy), and Siena University Hospital (Italy), were studied. Thirteen PBL, 7 EMPC and 9 BL (5 endemic) cases were used as discovery cohort for NGSbased miRNA profiling and EBV-latency assessment; 10 PBL, 10 EMPC and 10 BL cases were used as independent validation set of NGS analyses and were studied by qPCR and immunohistochemistry. The cases were reviewed by expert hemato-pathologists and diagnoses were confirmed by morphology on histological slides stained with haematoxylin and eosin (H\&E) and Giemsa, and by immunophenotyping, according to the WHO $[1,14]$. The following clinical data were collected: age, sex, disease location at presentation and an associated context of immunodeficiency.

Immunohistochemical studies were performed on representative paraffin sections from each case as previously reported [7, 15]. A large panel of antibodies (CD20, PAX-5, CD38, CD138, CD79a, CD10, BCL6, BCL2, MUM1, CD56, Igk, Igl) was applied to assess plasma cell differentiation. MYC protein expression and the proliferative index (by Ki-67) was evaluated. To rule out a diagnosis of ALK-positive large B-cell lymphoma or diffuse large B-cell lymphoma arising in multicentric Castlemann disease, immunohistochemical evaluation of ALK protein and HHV-8 was checked as well.

We studied the latency type of EBV by assessing the expression of some latent (EBNA-1, EBNA2, EBNA-3, LMP-1, LMP-2A) and lytic proteins (EA-D, EA-R, BZLF1/ZEBRA, BLLF1/gp350) by immunohistochemistry. In situ hybridization for the non-coding EBERs was carried out in each sample as previously described [7]. A control slide, prepared from a paraffin-embedded tissue block containing metastatic nasopharyngeal carcinoma in a lymph node, accompanied each hybridization run.

In addition, the expression level of the proteins (adipophilin, PPARGC1A, FOXK2, PBX2, and PTEN) encoded by the genes found to be potentially targeted by the cellular and viral miRNA deregulated in the studied tumors was checked in the different primary tumors to highlight their positive or negative regulation by miRNA [16]. Differences in protein expression between cases were analyzed using the Chi-square test with $p<0.05$ being 
statistical significant.

For all the stains, negative controls were obtained for each case by replacing the specific antibody with non-immune serum immunoglobulins at the same concentration as the primary antibody. Sections of reactive lymphoid tissue and of lymph node with infectious mononucleosis were used as positive control for plasma cell differentiation and EBV latency type and lytic phase.

FISH analysis for MYC rearrangements was performed using both dual-fusion probes and split-signal probes for IGH and IGL loci as well as an LSI IGH/ MYC CEP 8 Tri-color dual-fusion probe (Vysis, Abbott Molecular IL, USA) [15].

All the clinical, morphological, immunophenotypical and cytogenetic features of the discovery cohort patients are summarized in Table 1.

\section{MicroRNA profiling}

Total RNA was extracted from formalyn-fixed paraffed-embedded (FFPE) sections of primary tumors and reactive lymph nodes using the FFPE miRNA Easy kit (Qiagen, CA), according to the manufacturer's instructions. Small RNA libraries were prepared from $1 \mu \mathrm{g}$ of an high-quality RNA (RNA Integrity Number $\geq 8$ ) with the TruSeq Small RNA kit (Illumina). 1x36 sequencing was performed on the Illumina MiSeq platform. 36bp length raw sequences were demultiplexed using the Illumina pipeline CASAVA v1.8. A quality check of the run experiment was performed by FastQC (http://www. bioinformatics.babraham.ac.uk/projects/fastqc). Low quality reads and adpater sequences were trimmed off using Trimmomatic [17]. The high quality reads, with a length of 17-36-bp were clipped and subsequently aligned to the latest mirBase release (v 21 July 2014) [18] by Novoalign (http://www.novocraft.com) MiRNA expression profiles were built by calculating the sum of read counts for each miRNA, according to the alignment criteria. Variance-stabilizing transformed count data were used to build an Euclidean distance matrix, followed by hierarchical clustering analysis to study the intrasamples correlations. miRNA differential expression analysis was performed using Bioconductor's package DESeq [19]. The obtained read counts for each identified miRNA were first normalized by scaling for library size factors in order to deal with variation among samples. The differential expression values were estimated using a negative binomial distribution model and local regression to estimate the relationship between the dispersion and the mean of each miRNA. Raw values were considered as statistically significant for $p<0.05$. Following alignment and normalization, the data were analyzed using GeneSpring GX12 as previously described [20-25], by applying Pearson correlation (for unsupervised clustering) and two-tails Student t-test with $\mathrm{p}$-value $<0.05$ and fold change $>2$ (for supervised clustering). The experimentally validated targets for the viral and cellular miRNA were searched in public databases. For the viral miRNA, we used VIRmiRNA (http://crdd.osdd.net/servers/virmirna/) [26]; for the cellular miRNA, miRWalk 2 (http://zmf.umm. uni-heidelberg.de/apps/zmf/mirwalk2/) [27, 28]. Only proved direct interaction were then considered. Pathway analysis was performed by Gene Set Enrichment Analysis (GSEA) [29].

\section{Reverse transcription-quantitative PCR (RT- qPCR)}

The expression of EBV-encoded genes (EBNA-1, EBNA-2, LMP-1, LMP-2, EBNA-3, BZLF-1 BMRF-1/ $E a-D, \quad B H R F-1 / E a-R, \quad$ BLLF1/gp350) characterizing the different latency programs, has been investigated by RT-qPCR using the QuantiTect SYBR Green PCR Kit (Qiagen, CA). The stably expressed housekeeping gene hypoxanthine-guanine phosphoribosyltransferase (HPRT) was used as an endogenous control and reference gene for relative quantification of each target gene. The relative expression is expressed as $2^{\Delta \mathrm{Ct}}$, where $\Delta \mathrm{Ct}$ is defined as the difference in mean cycle thresholds of the gene of interest and HPRT.

The expression level of differentially expressed human (hsa-miR1304-3p, hsa-miR148a-3p, hsa-miR148a$5 \mathrm{p}$, hsa-miR192-5p) and viral miRNA (EBV-miR-BART10-5p, EBV-miR-BART-18-5p, EBV-miR-BART-19-3p), selected based on the score in differential analysis and the known biological relevance, was evaluated by using Taqman probes specific for each miRNA and for RNU6B (the endogenous control) (Apllied Viosystems, Applera, Italy). The relative expression of cellular miRNA was calculated using the $2^{-\Delta \Delta c t}$ formula; for viral miRNA the following formula was adopted: $2^{-\Delta c t}[30]$. Primers used for qPCR analyses are detailed in Table 2.

\section{Diagnostic accuracy evaluation}

A diagnostic accuracy analysis was designed to test the ability of a newly developed molecular classifier (in particular of the 4-miRNAs based classifier) to discriminate PBL and EPCM. Calculations of sensitivity, specificity, positive predictive value, negative predictive value, positive and negative likelihood ratio, and odd ratio were made by CATmaker software (Centre for Evidence Based Medicine, Oxford University, http://www.cebm. net). The study was conducted according to the STARD guidelines for biomarker testing (Supplementary Table 1).

\section{Author contribution}

The authors contributed in the following way: MRA, PPP and LL wrote the paper; PSV, SS, GLB, AG, 
SA provided materials; LM, MP, SG, MN carried out the experiments; MRA, LM, SL, PPP and LL discussed and interpreted the results. PPP and LL conceived the study. All the authors read and approved the final manuscript.

\section{ACKNOWLEDGMENT}

The Authors would like to thank Professor Giulia De Falco (Queen Mary University of London, UK), Professor Pankaj Trivedi (University "la Sapienza", Rome, Italy) and Professor Davide Gibellini (University of Verona, Italy) for providing their expertise in fruitful discussion.

\section{CONFLICTS OF INTEREST}

The authors declare no competing interests.

\section{FUNDING}

This work was supported BolognAIL, AIRC IG 2013 N.14355/Prof. Piccaluga, RFO (Prof. Piccaluga), and FIRB Futura 2011 RBFR12D1CB (Prof. Piccaluga).

\section{REFERENCES}

1. Stein H, Harris NL, Campo E. (2008). Plasmablastic lymphoma. In: Swerdlow S, Campo E, Harris NL, Jaffe ES, Pileri SA, Stein H, Thiele J, Vardiman J, eds. WHO Classification of Tumors of the Hematopoietic and Lymphoid Tissue. (Lyon.: IARC. ), pp. 262-4.

2. Taddesse-Heath L, Meloni-Ehrig A, Scheerle J, Kelly JC, Jaffe ES. Plasmablastic lymphoma with MYC translocation: evidence for a common pathway in the generation of plasmablastic features. Modern Pathology. 2010; 23: 991.

3. Castillo JJ, Bibas M, Miranda RN. The biology and treatment of plasmablastic lymphoma. Blood. 2015; 125: 2323-30

4. Vockerodt M, Yap LF, Shannon-Lowe C, Curley H, Wei W, Vrzalikova K, Murray PG. The Epstein-Barr virus and the pathogenesis of lymphoma. The Journal of pathology. 2015; 235: 312-22.

5. Laurent C, Fabiani B, Do C, Tchernonog E, Cartron G, Gravelle P, Amara N, Malot S, Palisoc M, Copie-Bergman C. Immune-checkpoint expression in Epstein-Barr virus positive and negative plasmablastic lymphoma: a clinical and pathological study in 82 patients. Haematologica. 2016; 101:976-84.

6. Ma SD, Hegde S, Young KH, Sullivan R, Rajesh D, Zhou Y, Jankowska-Gan E, Burlingham WJ, Sun X, Gulley ML. A new model of Epstein-Barr virus infection reveals an important role for early lytic viral protein expression in the development of lymphomas. Journal of virology. 2011; 85: 165-77.

7. Abate F, Ambrosio MR, Mundo L, Laginestra MA, Fuligni
F, Rossi M, Zairis S, Gazaneo S, De Falco G, Lazzi S, Bellan C, Rocca BJ, Amato T, et al. Distinct Viral and Mutational Spectrum of Endemic Burkitt Lymphoma. PLoS Pathog. 2015; 11: e1005158.

8. Montes-Moreno S, Martinez-Magunacelaya N, ZecchiniBarrese T, de Villambrosía SG, Linares E, Ranchal T, Rodriguez-Pinilla M, Batlle A, Revert-Arce JB, Almaraz C. Plasmablastic lymphoma phenotype is determined by genetic alterations in MYC and PRDM1. Modern Pathology. 2017; 30: 85-94.

9. Ambrosio MR, Piccaluga PP, Ponzoni M, Rocca BJ, Malagnino V, Onorati M, De Falco G, Calbi V, Ogwang $\mathrm{M}$, Naresh KN. The alteration of lipid metabolism in Burkitt lymphoma identifies a novel marker: adipophilin. PloS one. 2012; 7: e44315.

10. Schuhmacher M, Kohlhuber F, Hölzel M, Kaiser C, Burtscher H, Jarsch M, Bornkamm GW, Laux G, Polack A, Weidle UH. The transcriptional program of a human B cell line in response to Myc. Nucleic acids research. 2001; 29: 397-406.

11. Eberlin LS, Gabay M, Fan AC, Gouw AM, Tibshirani RJ, Felsher DW, Zare RN. Alteration of the lipid profile in lymphomas induced by MYC overexpression. Proceedings of the National Academy of Sciences. 2014; 111: 10450-5.

12. Altman N, Krzywinski M. Points of significance: P values and the search for significance. Nature Methods. 2017; 14: 3-4.

13. Daskalogianni C, Pyndiah S, Apcher S, Mazars A, Manoury B, Ammari N, Nylander K, Voisset C, Blondel M, Fåhraeus R. Epstein-Barr virus-encoded EBNA1 and ZEBRA: targets for therapeutic strategies against EBV-carrying cancers. The Journal of pathology. 2015; 235: 334-41.

14. Leoncini L, Raphaël M, Stein H, Harris NL, Jaffe ES, Kluin PM. Burkitt lymphoma. In: Swerdlow S, Campo E, Harris NL, Jaffe ES, Pileri SA, Stein H, Thiele J, Vardiman J, eds. WHO Classification of Tumors of the Hematopoietic and Lymphoid Tissue. (Lyon.: IARC. ). 2008; pp. 262-4.

15. De Falco G, Ambrosio MR, Fuligni F, Onnis A, Bellan C, Rocca BJ, Navari M, Etebari M, Mundo L, Gazaneo S, Facchetti F, Pileri SA, Leoncini L, et al. Burkitt lymphoma beyond MYC translocation: N-MYC and DNA methyltransferases dysregulation. BMC Cancer. 2015; 15: 668.

16. Ambrosio MR, Navari M, Di Lisio L, Leon EA, Onnis A, Gazaneo S, Mundo L, Ulivieri C, Gomez G, Lazzi S. The Epstein Barr-encoded BART-6-3p microRNA affects regulation of cell growth and immuno response in Burkitt lymphoma. Infectious agents and cancer. 2014; 9: 12.

17. Bolger AM, Lohse M, Usadel B. Trimmomatic: a flexible trimmer for Illumina sequence data. Bioinformatics. 2014; 30: 2114-20.

18. Kozomara A, Griffiths-Jones S. miRBase: annotating high confidence microRNAs using deep sequencing data. Nucleic Acids Res. 2014; 42: D68-73. 
19. Anders S, Huber W. Differential expression analysis for sequence count data. Genome Biol. 2010; 11: R106.

20. Navari M, Fuligni F, Laginestra MA, Etebari M, Ambrosio MR, Sapienza MR, Rossi M, De Falco G, Gibellini D, Tripodo C, Pileri SA, Leoncini L, Piccaluga PP. Molecular signature of Epstein Barr virus-positive Burkitt lymphoma and post-transplant lymphoproliferative disorder suggest different roles for Epstein Barr virus. Front Microbiol. 2014; 5: 728 .

21. Laginestra MA, Piccaluga PP, Fuligni F, Rossi M, Agostinelli C, Righi S, Sapienza MR, Motta G, Gazzola A, Mannu C, Sabattini E, Bacci F, Tabanelli V, et al. Pathogenetic and diagnostic significance of microRNA deregulation in peripheral T-cell lymphoma not otherwise specified. Blood Cancer J. 2014; 4: 259.

22. Navari M, Etebari M, De Falco G, Ambrosio MR, Gibellini D, Leoncini L, Piccaluga PP. The presence of EpsteinBarr virus significantly impacts the transcriptional profile in immunodeficiency-associated Burkitt lymphoma. Front Microbiol. 2015; 6: 556.

23. Hartigan JA. Clustering Algorithms. John Wiley \& Sons, Inc, New York. 1975: 351 pp.

24. Eisen MB, Spellman PT, Brown PO, Botstein D. Cluster analysis and display of genome-wide expression patterns. Proc Natl Acad Sci U S A. 1998; 95: 14863-8.

25. Piccaluga PP, Navari M, De Falco G, Ambrosio MR, Lazzi S, Fuligni F, Bellan C, Rossi M, Sapienza MR, Laginestra MA. Virus-encoded microRNA contributes to the molecular profile of EBV-positive Burkitt lymphomas. 2016; 7: 22440. http://doi.org/10.18632/oncotarget.4399.
26. Qureshi A, Thakur N, Monga I, Thakur A, Kumar M. VIRmiRNA: a comprehensive resource for experimentally validated viral miRNAs and their targets. Database (Oxford). 2014; 2014.

27. Dweep H, Gretz N, Sticht C. miRWalk database for miRNA-target interactions. Methods Mol Biol. 2014; 1182: 289-305.

28. Dweep H, Sticht C, Pandey P, Gretz N. miRWalk--database: prediction of possible miRNA binding sites by "walking" the genes of three genomes. J Biomed Inform. 2011; 44: 839-47.

29. Subramanian A, Tamayo P, Mootha VK, Mukherjee S, Ebert BL, Gillette MA, Paulovich A, Pomeroy SL, Golub TR, Lander ES, Mesirov JP. Gene set enrichment analysis: a knowledge-based approach for interpreting genome-wide expression profiles. Proc Natl Acad Sci U S A. 2005; 102: 15545-50.

30. Zhang G, Zong J, Lin S, Verhoeven RJ, Tong S, Chen Y, Ji M, Cheng W, Tsao SW, Lung M. Circulating EpsteinBarr virus microRNAs miR-BART7 and miR-BART13 as biomarkers for nasopharyngeal carcinoma diagnosis and treatment. Int J Cancer. 2015;136:E301-12. 